\title{
Irrupción de radio y divulgación en el aula para promover las vocaciones científicas en primaria
}

\author{
Daniel Martín-Pena iD \\ Departamento de Información y Comunicación. Universidad de Extremadura. Badajoz. España. \\ danielmartin@unex.es \\ Macarena Parejo Cuellar \\ Departamento de Información y Comunicación. Universidad de Extremadura. Badajoz. España. \\ macarenapo@unex.es \\ Agustín Vivas Moreno \\ Departamento de Información y Comunicación. Universidad de Extremadura. Badajoz: España. \\ aguvivas@unex.es
}

[Recibido: 23 abril 2020. Revisado: 10 junio 2020. Aceptado: 13 junio 2020]

\begin{abstract}
Resumen: Las actividades de divulgación de carácter proactivo aumentan el interés de los jóvenes por la ciencia y la tecnología. Máxime cuando las mismas se conjugan con una herramienta de enorme poder pedagógico como es la radio. A lo largo de un año, se ha llevado a cabo una experiencia con un millar de estudiantes de primaria de Extremadura denominada Ratones de Laboratorio. A partir del acercamiento de la labor científica, a través de talleres y programas radiofónicos, se ha tratado de medir los cambios de percepción experimentados en estos estudiantes. Para extraer los resultados, este estudio se ha basado en una experiencia real desarrollada en los propios colegios. A través de una herramienta metodológica basada en encuestas, al inicio y al final del desarrollo, se ha podido valorar y medir que la percepción de estos dos conceptos varía positivamente entre los niños participantes. Manifiestan que su interés por la ciencia ha aumentado y sus expectativas profesionales cambian hacia estudios más vinculados con las materias aprendidas durante la ejecución de la actividad. Del mismo modo, varían su impresión acerca del papel de los científicos en la sociedad.
\end{abstract}

Palabras clave: Divulgación científica; Radio; Educación Primaria; Extremadura; Vocaciones científicas.

Promoting STEM Careers in the primary school classroom through Radio and Scientific Outreach

Abstract: Proactive outreach activities increase young people's interest in science and technology. Especially when combined with a tool of enormous pedagogical power such as radio. Over the course of a year, an experience has been carried out with a thousand primary school students from Extremadura called «Laboratory mice». From the perspective of scientific work, through workshops and radio programs, attempts have been made to measure the changes in the perception experienced in these students. To extract the results, this study has been based on a real experience developed in the schools themselves. Using a methodological tool based on surveys at the beginning and at the end of development, it has been possible to evaluate and measure that the perception of these two concepts varies positively among the participating children. They affirm that their interest in science has increased and their professional expectations change towards studies more related to the topics learned during the execution of the activity. Similarly, they vary their impression of the role of scientists in society.

Keywords: Scientific dissemination; Radio; Primary education; Extremadura; Scientific vocations.

Para citar este artículo: Martín-Pena D., Parejo Cuellar M., Vivas Moreno A. (2020) Irrupción de radio y divulgación en el aula para promover las vocaciones científicas en primaria. Revista Eureka sobre Enseñanza y Divulgación de las Ciencias 17(3), 3205. doi: 10.25267/Rev_Eureka_ensen_divulg_cienc.2020.v17.i3.3205

\section{La promoción de vocaciones científicas desde edades tempranas}

Iniciativas como las ferias y semanas de la ciencia y la tecnología, los campus científicos y tecnológicos de verano, el fin de semana científico o la noche de los investigadores sirven para

\author{
Revista Eureka sobre Enseñanza y Divulgación de las Ciencias \\ Universidad de Cádir. APAC-Eureka. ISSN: 1697-011X \\ bttp:/ / dx.doi.org/10.25267/Rev_Eureka_ensen_divulg_cienc.2020.v17.i3.3205 \\ http:/ / reuredc.uca.es
}


despertar las vocaciones científicas en España. Así lo pone de manifiesto el estudio titulado ¿Cómo podemos estimular una mente científica? y realizado por la Fundación Española para la Ciencia y la Tecnología (FECYT) y la Obra Social La Caixa (2015). Este informe elaborado a partir de los datos obtenidos durante dos cursos escolares y que ha contado con la participación de más de 2.500 estudiantes de ESO- refleja que, de forma general, las acciones de divulgación aumentan el interés de los jóvenes en estudiar ciencia o tecnología.

Desde nuestra propia óptica, esto es así porque en estas actividades los jóvenes no suelen permanecer como sujetos pasivos de la actividad. Muy al contrario, en estos contextos, los pequeños se convierten, en la mayoría de las ocasiones, en los verdaderos protagonistas de la acción científica. Y esto es realmente importante a la hora de erradicar y desdibujar ciertos estereotipos culturales y mejorar su autoestima.

Demostrarse a ellos mismos que también pueden y que la ciencia no es algo que esté fuera de su alcance, y de su capacidad e intelecto, es fundamental para fomentar y despertar esas vocaciones. Al fin y al cabo, que sientan que las STEM (Science, Technology, Engineering \& Mathematics) no son necesariamente difíciles y que pueden convertirse en una opción profesional de futuro altamente satisfactoria (Balba 2018).

Es cierto que mejorar la percepción que sobre la ciencia tienen los pequeños de la casa es un fenómeno complejo donde entran en juego numerosos factores que serán los que determinen que en un futuro los estudiantes elijan profesiones que consideran que encajan con su forma deser (Holland 1985), tales como el contexto familiar, la autoestima, los métodos de enseñanza., etc. Sin embargo, estas actividades de las que se viene hablando pueden ayudarles a reconocer en ellos mismos unas competencias que incluso habían sido poco fomentadas por sus principales prescriptores: profesores y padres o tutores, estableciendo de esta forma un diálogo que permita al alumnado identificarse con las carreras científico-tecnológicas.

De hecho, por ejemplo, condicionados por sus propias creencias culturales adquiridas, los chavales en edad escolar pueden llegar incluso a verse condicionados por el estereotipo que tienen diseñados en sus mentes acerca de los científicos y el trabajo en entornos científicos. Y, como advierte el Informe PISA (2015) aunque parezca mentira esto puede hacer que algunos estudiantes desistan de continuar su dedicación a las ciencias. Precisamente por ello, «además de desmontar los estereotipos de género, los padres y los profesores pueden promover la participación de los estudiantes en las actividades científicas, ayudándoles a tomar conciencia de todas las oportunidades laborales que se abrirían para ellos con una buena formación en ciencia y tecnología» (PISA 2015 p. 6).

Paralelamente, junto a los contextos familiares serán fundamentales los escenarios diseñados dentro del aula, pues uno de los aspectos que más influyen, y que deben tenerse muy presentes a la hora de estudiar ciencias, son las emociones. Y muchas veces dentro del aula las mismas, respecto a las enseñanzas científico-técnicas, no son las mejores.

En este sentido, un estudio llevado a cabo por Sánchez-Martín, Cañada y Dávila (2017) -entre un grupo de 60 alumnos- pone de manifiesto que no todas las técnicas empleadas sirven para provocar este acercamiento a partir de la experiencia emocional. De sus averiguaciones, los autores pudieron extraer conclusiones interesantes como que «con el empleo de metodologías tradicionales, como la exposición oral, los estudiantes no consiguen reportar un nivel interesante de sorpresa, algo que sí hacen con metodologías aplicadas tipo bands on o simplemente enriqueciendo la exposición con herramientas de gamificación» (Sánchez-Martín Cañada y Dávila 2017 p. 1901). De alguna manera, esta reflexión parece haber calado en la sociedad a través de la puesta en marcha de acciones tanto regladas como de índole más lúdica y desenfadadas como las que eran citadas anteriormente. 
Muestra de ello es que ha habido un cambio de mentalidad. Es más, podemos decir que esta trasformación se ha llegado incluso a traducir también en cifras y datos objetivos, si nos referimos, por ejemplo, a estudios tan importantes y mediáticos como el Informe PISA.

Es significativo que, según el último Informe del Programa Internacional para la Evaluación de Estudiantes, que fue presentado en 2015, España se situaba por primera vez en la media, de los 34 países de la OCDE, más avanzados del mundo tanto en lecturas como en ciencias y casi también en matemáticas.

Es cierto que hasta ahora, y según se recoge en este último informe PISA, con frecuencia en las escuelas «suelen verse las ciencias como el primer tramo de un embudo (lleno de fugas) que terminará por seleccionar a quienes trabajarán como científicos o ingenieros [...] Por ello, el colegio debe presentar las ciencias de manera más positiva, quizá como un trampolín hacia nuevas fuentes de disfrute e interés». La solución, por lo menos una de ellas, sería aumentar la exposición temprana a una enseñanza científica de calidad en las escuelas.

Es cierto que tradicionalmente como recuerdan algunos autores ha sido habitual considerar el tramo de los 14-16 años como el momento más propicio para mejorar el interés por la ciencia (Bogdan y Greca 2016). Sin embargo, investigaciones realizadas en los últimos años ponen el foco en la necesidad de iniciar estas acciones vocacionales en las primeras etapas de la vida del niño (Rocard 2007). Fernández Pinto y Muñiz (2018 p. 285) refrendan esta teoría y propone que estas acciones «deberían iniciarse en la etapa de primaria, donde se obtiene un efecto más positivo, y prolongarse e intensificarse en educación secundaria».

En ello coinciden otros autores que incluso se atreven a proponer un nuevo modelo interdisciplinar de educación basado en la indagación. El mismo se basa en cinco grandes fases diferenciadas (Bogdan y Greca 2016):

1. El docente contextualiza a través de un problema de ingeniería.

2. Se procede a una indagación guiada a través de instrumentos tecnológicos que ayudan al diseño de experiencias e interpretación de datos.

3. En este proceso de indagación los estudiantes deben debatir los resultados obtenidos y proponer nuevas preguntas investigables necesarias para la resolución del problema inicial (incluyendo el planteamiento de hipótesis, diseño del plan de experimentación, y la forma de registrar los datos).

4. Los estudiantes plantean sus indagaciones abiertas teniendo como apoyo las referencias estándar de la indagación guiada, lo que les permite explorar de una manera más didáctica los contenidos específicos del temario, y conectar sus nuevas experiencias con los conceptos abstractos para así resolver el problema inicial

5. El maestro propone una posible aplicación tecnológica del descubrimiento, siendo ésta la evaluación del trabajo realizado.

La alfabetización científica y las metodologías empleadas para calar en el contexto escolar son, por lo tanto, fundamentales en todas las etapas escolares y más allá de la forma de enseñanza personal de cada docente, lo cierto es que la confección de una hoja de ruta programada, con actividades organizadas de manera interinstitucional, en colaboración en este caso con el gran centro del conocimiento, puede contribuir a revertir esa huida sistemática que muchos protagonizan. 


\section{La radio para el acercamiento de la ciencia a los escolares}

A la hora de hablar de promociones científicas desde edades tempranas no podemos perder de vista las posibilidades que brinda la radio educativa, dentro del aula como metodología innovadora en el acercamiento de la ciencia y la tecnología.

Como algunos autores defienden, el medio radiofónico es, sin lugar a dudas, una herramienta de enorme valía para educar y enseñar. «Las experiencias en países del Tercer Mundo en programas de alfabetización [...] muestran que la radio es un medio magnífico y tiene un gran papel que desempeñar en este campo» (Rodero 1997 p. 5).

De hecho, algunos expertos destacan sus posibilidades por encima de otros recursos audiovisuales como la televisión. En cualquier caso, el medio radiofónico es uno de los que mayor fuerza educativa posee y presenta enormes posibilidades dentro del aula (Martín Parejo y Vivas 2018). De hecho, para autores como Muñoz (1994) la radio en el entorno educativo posee muchas ventajas. Entre ellas, el hecho de que el profesor cuente con mayor atención del mensaje por parte del alumno-radioyente, la materia didáctica se puede adaptar al medio y la radio invita a la repetición de ideas, términos y conceptos que favorece la retentiva y asimilación.

Más allá de todo esto, la radio educativa es una de las herramientas que mayor capacidad de integración posee, entre otras razones, por sus posibilidades de trabajo colaborativo y de aprendizaje. Algo a lo que hay que unir que es el medio y recurso tecnológico más accesible y económico y esto es importante para poner en marcha un proyecto radiofónico en el ámbito educativo donde tampoco se cuenta con grandes presupuestos.

Todas estas características lo hacen propicio para ser trabajado desde el aula escolar (Carram et al. 2006). Para Jiménez (2001) la radio es el medio más enriquecedor desde el punto de vista pedagógico, y el que mayores posibilidades educativas ofrece a través del trabajo de muchas competencias que fomentan los valores.

Además, poner en marcha un proyecto de radio educativa permite, según Villamizar (2002) abordar los temas ampliamente, desde distintos puntos de análisis y diversas ópticas, relacionar el trabajo escolar con el entorno más próximo, sobrepasando las paredes del aula, asumir el rol de una ciudadanía crítica y sumergirse en la práctica democrática, fomentando el diálogo y la confrontación de ideas. Mejora el rendimiento de los alumnos en áreas vitales como la lectura, la redacción, el análisis de temas, la expresión oral, la elaboración de síntesis, resúmenes y conclusiones. $\mathrm{Y}$, lo que es igualmente importante, al tener que trabajar en equipo y responsabilizarse de determinadas tareas radiofónicas vinculadas al conocimiento aprendido durante los talleres, los chavales desarrollan una seguridad hacia estas disciplinas que a largo plazo puede ayudarles a erradicar ciertos estereotipos en torno a la ciencia. Este proceso los activa, les exige una formación más completa, incrementando el alumno sus capacidades de tipo analítico, crítico, creativo o comunicativo.

Como señalan Martín-Pena, Parejo y Vivas (2018) la radio educativa es también sinónimo de motivación de alumnos, de cambios metodológicos adaptados a los nuevos tiempos donde priman las tecnologías y la información está al alcance con un solo clic. Y, todo ello supone una transmisión más efectiva de la educación en valores, una mayor participación del alumnado y una mejora considerable del ambiente del aula y de las relaciones interpersonales alumno-alumno y alumno-profesor, e incluso profesor-padres.

En este sentido, al hablar de radio educativa debemos entenderla casi como sinónimo de radio universitaria Martín-Pena, Parejo y Vivas (2016), al ser un instrumento comunicativo de la institución docente tiene una misión pedagógica y de transferencia del conocimiento para con 
la sociedad. Y es que como recuerda Segura (2014 p. 51) las emisoras universitarias más allá de ser un instrumento de aprendizaje práctico «para futuros profesionales del medio y de escenario de experimentación de nuevas o recuperadas tendencias radiofónicas, pero también como divulgador de las actividades del campus y vía de comunicación entre la comunidad universitaria que demanda más visibilidad para sus actividades». Y todo esto en parte es, como recuerdan Espino y Martín-Pena (2012), porque el desarrollo de Internet ha proporcionado a estas radios un nuevo sistema de dinamización social y cultural, para conectar mejor con los jóvenes, que encuentran más posibilidades de interactuar y tener una participación activa en este tipo de emisoras, participación que va más allá de las aulas y de los pasillos de sus facultades para salir a la sociedad en general.

De Lorenzo (2013) hace especial hincapié en las posibilidades que posee la radio universitaria en la divulgación de la ciencia. Máxime si se utiliza como un instrumento de formación para aprender haciendo. Siguiendo la famosa frase pronunciada por el político estadounidense Benjamín Franklin «Dime y lo olvido, enséñame y lo recuerdo, involúcrame y lo aprendo», Sanmartí (2007) recuerda que aquellos estudiantes que realizan actividades científicas no solo aprenden nuevas ideas, sino que desarrollan nuevas cualidades para llevar a cabo innovadoras formas de comunicación.

De ahí que, el maridaje ciencia y radio en el contexto escolar deba fomentarse en ese proceso de aprendizaje y de promoción de vocaciones científicas.

\section{El proyecto de divulgación científica «Ratones de Laboratorio: radio y ciencia en la escuela»}

Bajo esta premisa, la Universidad de Extremadura y la Fundación Universidad Sociedad pusieron en marcha un proyecto capaz de aunar ciencia y radio como fórmula para fomentar en el contexto escolar las vocaciones científicas.

Se trata de Ratones de Laboratorio, la iniciativa recibió el respaldo por su carácter innovador en la Convocatoria de Ayudas para el Fomento de la Cultura Científica, Tecnológica y de la Innovación de la Fundación Española para la Ciencia y la Tecnología, la FECYT.

En este proyecto, las entidades promotoras de la iniciativa partían de la idea de poner en marcha una iniciativa de carácter lúdica y pedagógica con la que promover la divulgación y el conocimiento científico-tecnológico de los estudiantes de educación primaria de los centros educativos de la región de Extremadura.

Pretendían lograrlo, sobre todo, a través del acercamiento de dos figuras fundamentales a los más pequeños: los medios de comunicación, en este caso la radio universitaria, y la figura del investigador, como fórmula de erradicación de ciertos estereotipos en torno a estas profesiones.

Como es lógico, más allá de estos dos objetivos generales y primigenios, la actividad pretendía, además, plantear y alcanzar una serie de objetivos específicos entre los que se encontraban: acercar la ciencia universitaria y la figura del científico a la sociedad infantil; despertar en los estudiantes el gusto por la ciencia a través de productos hechos en sus propios centros de trabajo; sacar al alumnado de la rutina del aula sin dejar de lado su formación, así como crear una red de colaboradores/maestros, científicos/divulgadores y comunicadores entre las universidades españolas y sus centros educativos, entre otros.

Lo más reseñable de esta iniciativa desde el punto de vista de la ejecución del proyecto fue su dinámica de trabajo. La misma ha estado basada en dos claros conceptos. De una parte, hacer de esta experiencia una oportunidad para la construcción colectiva del proyecto partiendo de 
la colaboración entre los maestros de los centros educativos extremeños y profesionales científicos de la universidad. De la otra, la filosofía del pro-común, poniendo en valor y divulgando el conocimiento de los expertos de la Universidad de Extremadura a partir de la puesta en común de todos los agentes implicados en el proceso.

De esta forma se ha trabajado a través de un protocolo muy delimitado. Partiendo del dato de que en cada nuevo taller han participado medio centenar de alumnos, puede decirse que cada nueva experiencia ha arrancado de la mano de los investigadores de la Universidad de Extremadura que, a través de breves charlas y sencillos experimentos, han explicado en primer lugar cuestiones científicas de la vida diaria y presentes en el currículo de los alumnos extremeños en esta etapa docente.

Más tarde, pero en el marco de la misma sesión iniciada por los científicos, los más pequeños han participado en la realización de un programa de radio donde se han enfrentado a retos importantes como poner a prueba los conocimientos adquiridos durante el taller. La comunicación oral ha jugado por tanto un papel importante a la hora de asentar los conocimientos adquiridos. Para desarrollar el proyecto, los colegios seleccionados han sido un total de 9 ( 7 de la provincia de Badajoz y 2 de la provincia de Cáceres). Y el listado de talleres desarrollados ha sido el que se detalla a continución.

Por una parte, los talleres para Segundo Ciclo de Primaria (tercer y cuarto curso): Los sentidos y los alimentos, Tu salud empieza por los pies, ¿Conciencia2? Recicla que algo queda y La vida en los charcos. Por otra, los talleres para Tercer Ciclo de Primaria (quinto y sexto curso): Concienciación ambiental, Internet de las cosas, Carrera de flechas y La magia de la catálisis. En total se han realizado 16 talleres. En cada centro educativo se han impartido 2 talleres (uno para el segundo ciclo de primaria y otro para el tercer ciclo).

De estos talleres se extrajeron los programas de radio, también 16, uno correspondiente a cada uno de los talleres. Además, posteriormente un jurado compuesto por periodistas, docentes, e investigadores de la región de Extremadura valoraron estos productos comunicativos creados por los propios participantes como ya se ha explicado ampliamente en base a una serie de criterios: nivel de conocimientos adquiridos, destrezas comunicativas, originalidad e ingenio de los participantes para responder a las preguntas, capacidad de síntesis o creatividad en la presentación del contenido.

Más allá de todo esto, el proyecto Ratones de Laboratorio obtuvo en el certamen Ciencia en Acción-Adopta una estrella en la categoría de Física y Sociedad una mención de honor. Además, fue seleccionado para participar en el certamen Science on Stage y ser expuesto a nivel europeo como ejemplo de una acción comunicativa innovadora realizada desde España en el fomento de las vocaciones científicas y la divulgación de la ciencia en edades tempranas.

\section{Metodología}

El proyecto Ratones de Laboratorio tenía como principal propósito fomentar las vocaciones científicas en edades tempranas. Sin embargo, su desarrollo y el contacto directo de los autores de este estudio con la población objeto de análisis, suponía también una oportunidad para conocer, desde una óptica investigadora, la efectividad que este tipo de acciones puede suponer a la hora de mejorar la percepción social de la ciencia. Por ello, desde un punto de vista metodológico el trabajo ha perseguido dos objetivos generales:

OG1: evaluar de manera sistemática y continuada las emociones sentidas por los estudiantes que se enfrentan a materias científicas.

OG2: identificar diferencias significativas en el rendimiento emocional de estos estudiantes en 
función de las metodologías docentes empleadas.

A partir de estos objetivos generales este análisis partía de dos hipótesis fundamentales que enumeramos a continuación:

H.1. El desarrollo de actividades de divulgación científica en edades tempranas ayuda a aumentar el interés por los aspectos vinculados por la ciencia y la tecnología.

H.2. El acercamiento de la ciencia y la tecnología al aula permite que el niño vea la figura del investigador desde una perspectiva de cercanía y se planté la posibilidad de ser uno ellos en el futuro.

Para alcanzar estos propósitos, el proyecto quedo dividido en dos etapas diferentes. El presente estudio recoge los resultados de la segunda fase de desarrollo del mismo (etapa donde se aplicó en instrumento metodológico).

Es preciso matizar que en una primera etapa, la de ejecución de las actividades como parte del trabajo de campo, se expuso la dinámica del proyecto, así como los productos generados en el aula por el alumnado (programas de radio, dinámicas de los talleres, etc.). Se pueden consultar los vídeos de los talleres en la web https://vimeo.com/channels/ratlaboratorio y escuchar los programas de radio en https://www.ivoox.com/podcast-ratoneslaboratorio_sq_f1302928_1.html (OndaCampus 2020). Dicho material constituye una fuente didáctica para aplicar hoy en día en el aula.

También, en esta primera etapa se diseñó la herramienta de investigación. El instrumento para medir las sensaciones despertadas a partir de la experiencia vivida en el aula que se escogió fue la encuesta. Fue la herramienta elegida, por cuanto se trata de un «método de investigación capaz de dar respuestas a problemas tanto en términos descriptivos como de relación de variables, tras la recogida de información sistemática, según un diseño previamente establecido que asegure el rigor de la información obtenida» (Buendía Colás y Hernández 1998 p. 120).

La encuesta elaborada fue consensuada y validada por un comité de expertos compuesto por profesionales de la comunicación, educadores sociales, divulgadores y docentes en edades tempranas. Una vez confeccionado, se estableció un plazo para recibir sugerencias y tras el mismo se procedió a variar la herramienta en base a las apreciaciones planteadas por cada uno de los expertos del panel.

Si bien es cierto que al término de la experiencia tanto a profesores como alumnos se les cuestionó por aspectos concretos de la organización de la actividad, fueron siete preguntas orientadas a dar respuesta a la investigación (OG1 y OG2/H.1 y H.2). Las cuestiones que se plantearon fueron las siguientes: ¿'Te gusta la ciencia?, ¿sueles participar en talleres donde te enseñen experimentos?, ¿valora de uno a cinco lo que te gusta la ciencia?, ¿qué asignatura te gusta más?, ¿qué es para ti un científico?, ¿qué quieres ser de mayor?, ¿te gustaría ser científico?

Se optó por una metodología de tipo cuantitativo, ya que existía la posibilidad de trabajar con una muestra amplia de sujetos para conocer el sentir colectivo de la efectividad de la acción. Además, dado el público al que iba dirigida la resolución de las respuestas -niños entre 8 y 12 años principalmente-, no se configuró un instrumento donde solo se solicitara que las respuestas se valoraran a través de la escala de Likert (Parejo 2015) y a través de medidas graduadas de 1 a 5 , siendo el 1 equivalente a «totalmente en desacuerdo» y el 5 a «totalmente de acuerdo», con un punto intermedio que es el 3. Del mismo modo, se plantearon preguntas dicotómicas con respuesta sí/no y otras de contestación libre.

Los cuestionarios fueron procesados en dos olas. Primeramente, fue respondido al inicio de la actividad y posteriormente, una vez concluidos los talleres científicos y elaborado el programa 
de radio. Respondieron la encuesta previa un total de 402 alumnos frente a las 375 encuestas finales que fueron respondidas por los alumnos una vez desarrolladas las actividades de radio y ciencia. Las preguntas planteadas en ambas oleadas se han caracterizado por su similitud. A fin de cuentas, el objetivo era extraer conclusiones acerca del cambio o no de comportamiento de los participantes tras su participación en esta actividad que pretende aunar formatos divulgativos y radiofónicos en el aula.

Tras la segunda oleada se recogieron y compararon en detalle todos los resultados de las encuestas procesadas en base a criterios morfológicos y de escala. En este sentido, consideramos importante que este tipo de proyectos contengan una parte de evaluación del resultado en cuanto a su uso como herramientas válidas que puedan promover las vocaciones STEM.

Sin lugar a dudas, configurar de esta forma el instrumento metodológico de este estudio permitió medir actitudes y conocer el grado de conformidad del encuestado con cualquier afirmación que se le propusiese, y eso es especialmente útil en situaciones como la que se planteaba en este trabajo, en las que se quiere que la persona matice su opinión. Con el hándicap, además, de las edades que presentaban los encuestados.

\section{Resultados}

Para este estudio se han gestionado un total de 779 cuestionarios respondidos por alumnado de tercero a sexto de primaria de colegios repartidos por toda la geografía extremeña.

De ellos, 402 corresponden a la primera oleada (5 \% tercero de primaria; $30 \%$ cuarto curso; $20 \%$ quinto curso y $45 \%$ de sexto de primaria) y 377 a la segunda fase del cuestionario $(15 \%$ tercer curso, $29 \%$ cuarto, $32 \%$ quinto y $24 \%$ de sexto de primaria).

\section{Gusto por la ciencia}

Una de las primeras preguntas planteadas a los pequeños es si les gustaba la ciencia. A través de cuestiones dicotómicas se les proponían cuatro niveles de respuesta: Sí, mucho; sí, aunque no mucho; no, aunque hay cosas que me gustan y no, no me gusta nada que tenga que ver con la ciencia.

En la figura 1, que recoge los resultados de la primera y de la segunda oleada, se pueden observar algunos datos significativos, como que ha aumentado en casi dos puntos el máximo interés manifestado, pasando de $49,3 \%$ a un $51,2 \%$.

También es de destacar que ha disminuido el porcentaje de los que manifestaban no tener interés ninguno en la ciencia (No, aunque hay cosas que me gustan ha pasado de 13,2 \% a $13,1 \%$ y no, no me gusta nada que tenga que ver con la ciencia ha variado de $3,7 \%$ a $2,4 \%$ en la segunda oleada y tras haber pasado por la actividad). 


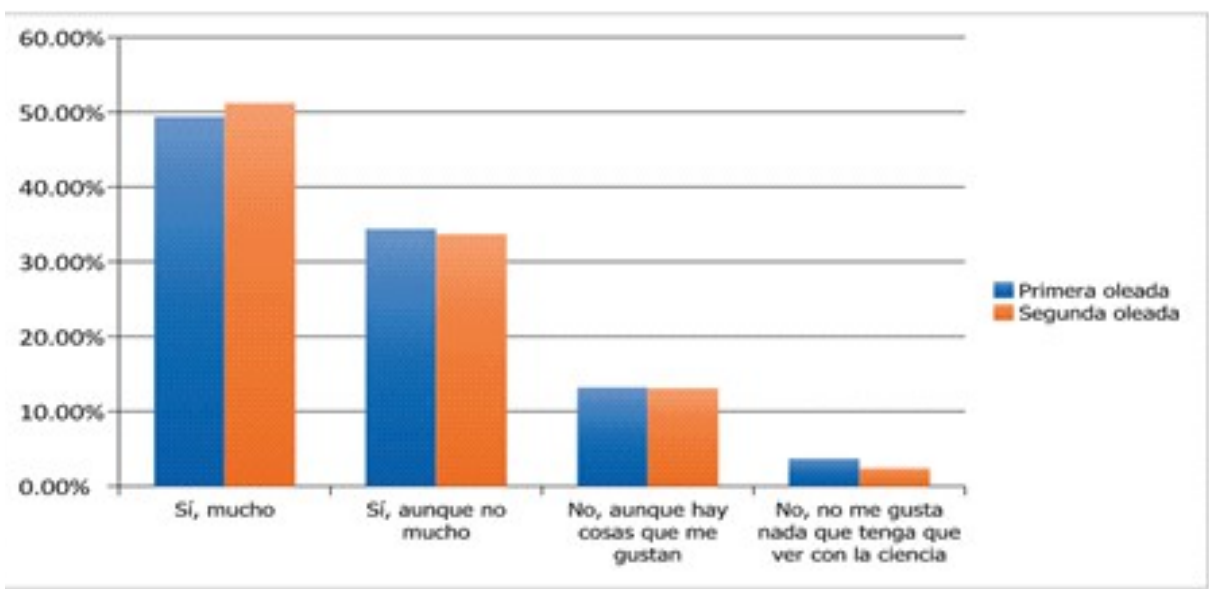

Figura 1. ¿'Te gusta la ciencia? Oleada 1 y 2.

\section{Participación en actividades de divulgación científica}

Otra de las cuestiones que se pretendía conocer era la asiduidad que los chavales tenían a actividades relacionadas con la divulgación científica. Para ello, se les preguntó directamente si solían participar en talleres donde les mostrasen experimentos.

Este planteamiento también fue lanzado en hasta dos ocasiones, ya que entendíamos que tras la actividad y al ser niños, aun en edades tempranas, podían entender mejor el planteamiento e incluso recordar algunas experiencias vividas que pudieran haber olvidado y que le viniera a la mente tras participar de los talleres preparados en el marco del proyecto.

En la figura 2 que se expone a continuación puede apreciarse que el porcentaje tanto en la primera como en la segunda oleada de chavales que afirma acudir con frecuencia es escaso (10\% en la primera oleada y $12,8 \%$ en la segunda). El grueso de respuestas se concentra entre el «no, aunque alguna vez»y «no, nunca» con 33 y $35 \%$ primera oleada y $39,30 \%$ y 21,90\%, respectivamente en la segunda.

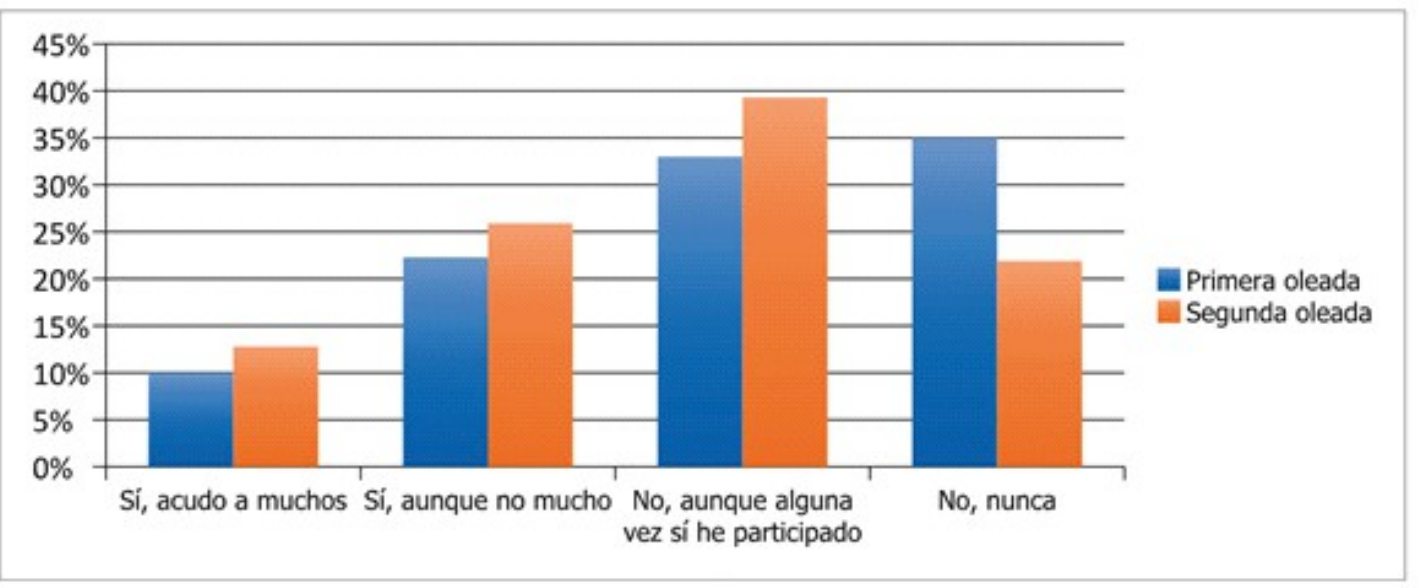

Figura 2. ¿Sueles par-ticipar en talleres don-de te enseñen experi-mentos? Oleada 1 y 2.

\section{Las asignaturas preferidas en el alumnado de primaria}

También se les pidió que indicaran, esta vez de forma libre y sin dar opción a respuestas cerradas, qué asignatura les gustaba más. La más nombrada en la primera oleada fue la Educación Física seguida de Matemáticas. Es reseñable indicar en este apartado de resultados obtenidos que en la segunda oleada las matemáticas fueron colocadas en la primera posición seguida de Educación Física. 
Sin embargo, y a pesar de que aparecen en la primera y segunda fase del cuestionario en este orden, lo cierto es que, analizando las respuestas tanto desde un punto de vista cualitativo como cuantitativo, no se aprecian cambios significativos en sus respuestas.

Tanto en la primera oleada como en la segunda, el $40 \%$ alumnos de primaria encuestados manifestaban que su materia favorita estaba vinculada directamente con las ciencias (física, biología, ciencias naturales, etc...). Ni aumentaba ni disminuía este porcentaje si se establecía una comparativa entre ambos cuestionarios.

\section{¿Qué es para ti un científico?}

Otra de las preguntas abiertas que se les sugirió es que describieran qué era para ellos un científico. En ese sentido, se puede apreciar que la descripción ofrecida por los chavales varía, extendiéndose tras la actividad. Así, si bien es cierto que las respuestas ofrecidas en la primera oleada son correctas, lo cierto es que el $100 \%$ de ellos parecía tener una idea demasiado limitada de lo que es un investigador. Algunas de las descripciones obtenidas la primera vez fueron las siguientes: una persona que hace experimentos; una persona que estudia cosas nuevas; una persona que realiza experimentos; una persona que hace ciencia; una persona que experimenta con cosas, etc.

Esta definición, sin embargo, se aprecia que incluye más detalles y es mucho más específica en las respuestas tras la realización de la actividad. Extraemos algunas de las respuestas que nos parecen representativas de este cambio de percepción registrado tras la experiencia: una persona que investiga y trata de inventar curas o cosas que nos beneficien a nosotros; un científico es una persona que realiza experimentos, que investiga cada vez más sobre los medicamentos u otro tipo de cosas; una persona que hace experimentos con animales, plantas y líquidos raros; una persona que nos ayuda a descubrir lo que aún no se conoce. Me encantan los científicos, etc.

Otro de los datos arrojados que han llamado la atención es el relativo a las profesiones. En una pregunta abierta en la que simplemente se le pregunta al alumno qué quiere ser de mayor el porcentaje de respuestas que nombran expresamente la palabra científico pasan de un 2 a un $5 \%$ lo que supone un avance algo significativo.

En la pregunta en la que se aprecian unos resultados más reseñables es en la última de las cuestiones comunes entre la primera y la segunda oleada. Y es que aquí se observa cómo los indecisos en plantearse ser científicos en el futuro en la primera fase del cuestionario pasan a responder afirmativamente que desean ser científicos el día de mañana en la segunda encuesta. Así lo podemos apreciar en la figura 3 que sigue a continuación.

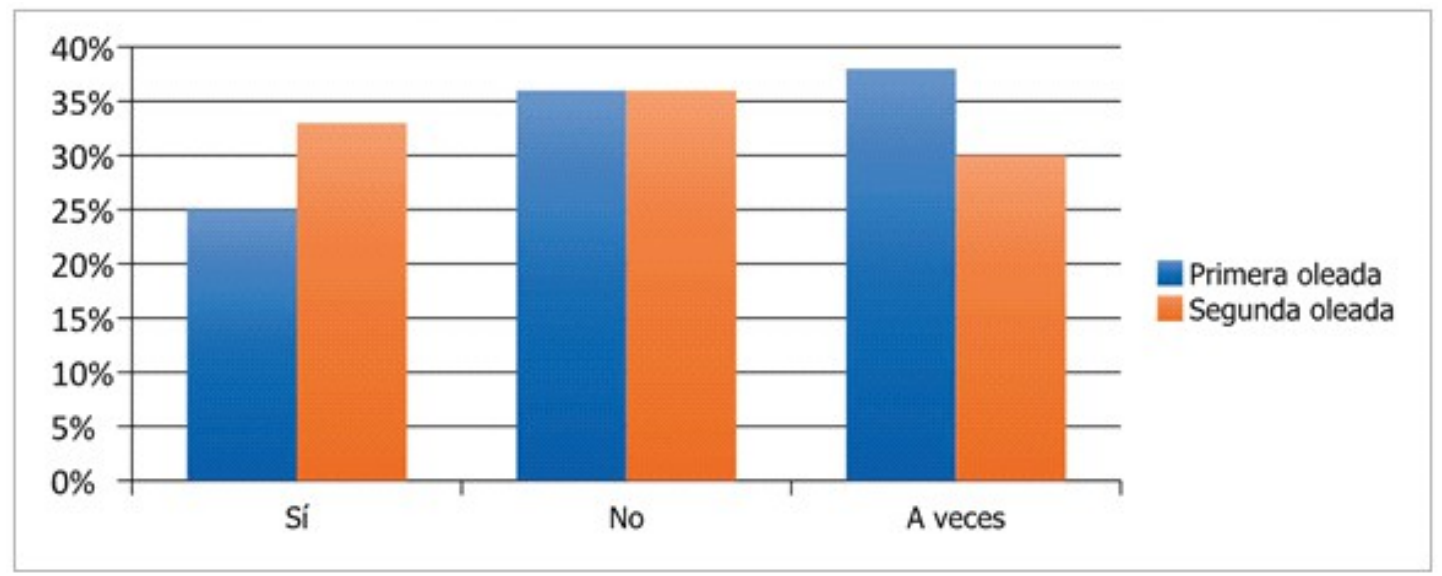

Figura 3. ¿'Te gusta-ría ser científico? Oleada 1 y 2. 
En ella se puede apreciar cómo la respuesta «sí quieren ser científicos de mayores» pasa del $25 \%$ al $33 \%$, justo el $8 \%$ que baja en porcentaje entre la primera y la segunda fase la respuesta «a veces pienso en convertirme en científico cuando sea mayor».

También llama la atención que el porcentaje de respuestas con "no me gustaría convertirme en científico de mayor" se mantiene intacto con un $36 \%$ en ambas ocasiones.

\section{Planteamiento de las acciones divulgativas de Ratones de Laboratorio}

A estos datos hay que unir las sensaciones que ha despertado en ellos el taller de ciencia y la experiencia radiofónica del proyecto Ratones de Laboratorio. En este sentido, el $90 \%$ de los participantes decían estar completamente de acuerdo con la forma de plantear la actividad. Además, cerca del $82 \%$ querría repetir la experiencia con mayor asiduidad. En la figura 4 vemos las respuestas contestadas a través de la escala Likert (siendo 5 la opción de repetir la experiencia muchas veces y 1 todo lo contrario).

Tras analizar los resultados de esta última cuestión, hemos observado que resulta muy positivo realizar actividades que acerquen el, a veces alejado, mundo universitario a los centros educativos de primaria, y así lo han transmitido los maestros. En este aspecto, hemos continuado desarrollando otro tipo de actividades que invitan a los niños a aprender de una manera divertida y diferente, podíamos decir que aprender jugando. De esta forma, hemos lanzado proyectos que prosiguen con esta línea de trabajo, pero aplicándole dosis de innovación que no deje de sorprender a los más pequeños, y que promuevan el contacto directo con los investigadores y los científicos.

\section{Conclusiones}

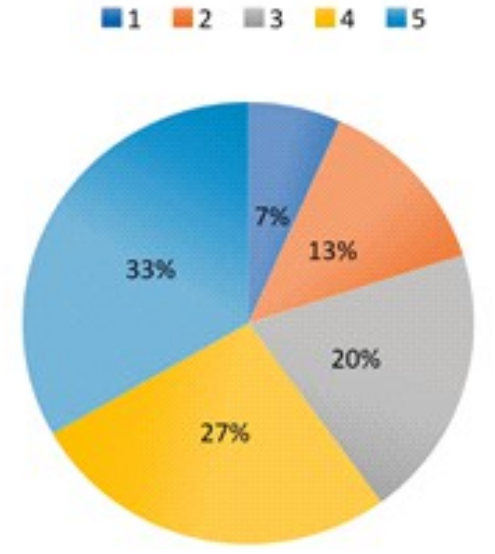

Figura 4. ¿Te gustaría repetir la experiencia de «Ratones de Laboratorio»?

Ratones de Laboratorio ha permitido aunar ciencia y radio en los centros educativos. Tanto por lo expuesto previamente por diversos autores citados a lo largo de este artículo como por la experiencia vivida durante el proyecto a través del contacto directo con los centros escolares, es posible llegar a la conclusión de que esta mezcla es un maridaje recomendable para promover las vocaciones científicas desde las primeras etapas escolares.

Sobre todo, porque en esta actividad se aprende haciendo. Los niños pueden llegar a convertirse en protagonistas de todas las acciones que engloba la iniciativa (experimentan en los talleres de ciencia y no son sujetos pasivos, son ellos mismos los que hacen el programa de radio, etc.).

Su carácter proactivo es importante del mismo modo por el acercamiento que se produce entre dos contextos en principio equidistantes, como son el universitario y el escolar.

La vinculación con la ciencia, a través de la participación en sencillos experimentos, donde la interacción del niño es una constante, contribuye, sin duda, a despertar el interés y mantener la motivación durante todo el proceso de enseñanza/aprendizaje.

Además, el hecho de que la actividad cuente con una segunda fase dentro del aula, esto es, la realización de los programas de radio, contribuye a que el alumno deba mantener la atención. En Ratones de Laboratorio los alumnos eran conocedores de que tras el taller debían trasladar lo aprendido a los micrófonos y esto, sin duda, mejoró la concentración prestada durante la actividad. 
A pesar de todo ello, y a la luz de los resultados obtenidos, sí que se aprecia que el cambio de percepción no es lo significativo que se esperaba inicialmente.

Si bien se partía de la hipótesis inicial de que H.1. El desarrollo de actividades de divulgación científica en edades tempranas ayuda a aumentar el interés por los aspectos vinculados por la ciencia y la tecnología, lo cierto es que debemos reconocer que dicha afirmación solo se cumple de forma parcial.

El porcentaje de respuestas afirmativas ante la pregunta ¿te gusta la ciencia? con un «sí, mucho» ha aumentado en un $2 \%$ tras la realización de la actividad. Este dato puede poner de manifiesto la idoneidad de estas acciones a la vez que plantea la necesidad de prolongarlas en el tiempo. Quizás, implantar acciones que se extiendan a lo largo del cuso académico sirvan para asentar las bases de esa promoción de las vocaciones que pretende desarrollarse en el seno de nuestra sociedad actual. Esta puede ser la fórmula para aumentar el porcentaje de niños que afirman sin ningún resquicio de duda que las materias científicas le interesan.

No obstante, este estudio puede servir para apoyar la opinión de expertos en la materia que afirman la necesidad de reforzar el acercamiento a la ciencia desde edades tempranas. En este sentido, sí que se aprecia, tras esta investigación, que actividades y proyectos de divulgación científica como el que aquí se ha expuesto sirven para lo que se afirma en la H.2 de nuestro estudio. Y es que el acercamiento de la ciencia y la tecnología al aula permite que el niño vea la figura del investigador desde una perspectiva de cercanía y se planté la posibilidad de ser uno ellos en el futuro.

Prueba de ello es aquellos que solo de vez en cuando se planteaban la posibilidad de ser en el futuro un científico habían cambiado tras la actividad su respuesta y ahora lo veían como una verdadera posibilidad. También las definiciones ofrecidas, donde en una primera oleada los niños hablan del investigador de forma genérica para posteriormente detallar algunas de sus misiones son clara muestra de ello.

Más allá de los números arrojados en las respuestas hay otros muchos aspectos reseñables que nos ha aportado la experiencia. Uno de ellos es el papel que juegan el centro y el docente como prescriptores de ciencia para los pequeños de la casa. Se ha observado cómo las generaciones de profesores más jóvenes son sobre todo los que más motivan al alumnado hacia estas materias.

Lógicamente el feeling del profesor hacia materias como la física, la química, la biología, etc...es determinante en la confección de un escenario favorable para el aprendizaje.

En Ratones de Laboratorio se ha podido apreciar que más allá de que los estudiantes estuvieran en su mayoría dispuestos a participar de más actividades similares a estos talleres de ciencia y radio, ha sido fundamental la actitud tomada por el docente. En aquellos casos en los que el profesor había trabajado previamente la temática con los alumnos la comunicación entre el binomio investigador-alumnado era mucho más fluido, como puede deducirse.

Por todo ello, si bien defendemos la bondad de este tipo de acciones puntuales como fórmula para mejorar la percepción de la ciencia de los más jóvenes de la casa y aprovechar las misiones de la radio universitaria para llevar a cabo esa transferencia del conocimiento que es misión de las universidades, de lo vivido y de los resultados obtenidos se deducen también otras necesidades.

- Por un lado, sería preciso diseñar programas regulares en el tiempo sobre divulgación científica para el alumnado de primaria (aunque esta reflexión sería extrapolable a otras etapas educativas que no han sido objeto de estudio en este trabajo). 
- Por otro lado, sería conveniente reforzar la formación de los profesores de primaria en divulgación científica. Enseñar a los docentes el enfoque de talleres dentro del aula o utilizar la radio como instrumento pedagógico para las STEM podría incrementar la presencia de la ciencia y la motivación por estas dentro del aula.

\section{Agradecimientos}

Este estudio, cuyo código ético y de buenas prácticas de publicación se adhiere a la Revista Eureka sobre Enseñanza y Divulgación de las Ciencias, ha sido cofinanciado con Fondos FEDER dentro del Plan Regional de I+D+I de la Consejería de Economía e Infraestructuras de la Junta de Extremadura para la ayuda para la realización de investigación, de divulgación y de transferencia del conocimiento por los Grupos de Investigación de Extremadura. Referencia Grupo (TIC013). Además de la ayuda de la Fundación Española para la Ciencia y la Tecnología para impulsar esta iniciativa a través la Convocatoria de Ayudas para el Fomento de la Cultura Científica, Tecnológica y de la Innovación de la Fundación Española para la Ciencia y la Tecnología, la FECYT.

\section{Referencias}

Balba C. (2018) Nos faltan vocaciones científicas. Fundación Aquae.

Bogdan T., Greca L. (2016) Modelo interdisciplinar de educación STEM para la etapa de Educación Primaria. $3^{\mathrm{r}}$ Simposio Internacional de Enseñanza de las Ciencias SIEC. Vigo: Universidad de Vigo.

Buendía L., Colás P., Hernández F. (1998) Métodos de Investigación en Psicopedagogía. Madrid: McGraw-Hill.

Carram M., Soria G., Llimós G., Borrastero C., Pérez Cuevas N. (2006) La radio en la escuela. ¿Sólo un medio para aprender más? Razón y Palabra 49, 131-143.

De Lorenzo I. (2013) Del artículo científico a las ondas: cómo el periodismo radiofónico universitario puede mediar entre la ciencia y la sociedad. pp. 106-120 en P. Contreras y M. Parejo (eds.) + Ciencia. Cómo trabajar la divulgación cientifica desde las radios universitarias. Salamanca: Comunicación Social.

Espino C., Martín-Pena D. (2012) Las radios universitarias, más allá de la radio. Las TIC como recursos de interacción radiofónica. Barcelona: Universitat Oberta de Catalunya.

FECYT (2015) ¿Cómo podemos estimular una mente cientifica? Estudio sobre vocaciones científicas?. Barcelona: Obra Social "La Caixa".

Fernández R., Pinto N., Muñiz M. (2018) ¿Mejoran los proyectos de divulgación con experimentación la actitud hacia las clases de ciencias? Revista de Educación 381, 285-308.

Holland J. L. (1985) Making Vocational Choices. A Theory of Vocational Personalities and Work Environments. Englewood Cliffs, NJ: Prentice-Hall.

Jiménez J. (2001) La radio a la escuela. Contextos educativos. Revista de Educación 4, 297-313.

Martín-Pena D., Parejo M., Vivas A. (2016) La radio universitaria: Gestión de la información, análisis y modelos de organización. Barcelona: Gedisa.

Martín-Pena D., Parejo M., Vivas C. (2018) Radio educativa para fomentar las vocaciones científicas. Index.comunicación 8(2), 229-254.

Muñoz J. J. (1994) Radio educativa (1 ${ }^{\mathrm{a}}$ ed.). Salamanca: Librería Cervantes.

OndaCampus (2020) Ratones de laboratorio. Badajoz: Proyecto Ratones de Laboratorio.

Rocard M., Rocard M., Csermely P., Jorde D., Lenzen D., Walberg-Henriksson H., Hemmo V. (2007) Science Education Now: A renewed Pedagogy for the Future of Europe. Brussels: Directorate General for Research, Science, Economy and Society. 
Parejo M. (2015) Los gabinetes de comunicación de las Universidades españolas: propuesta de modelo y análisis de las salas de prensa virtuales universitarias (Tesis Doctoral). Badajoz: Universidad de Extremadura.

PISA (2015) Informe español. Programa para la Evaluación Internacional de los alumnos. Madrid: Servicio Publicaciones MECD.

Sánchez-Martín J., Cañada F., Dávila M. A. (2017) Hacia una medición emocional continua. Lo que sienten los estudiantes del grado en Educación Primaria en asignaturas de ciencia básica. X Congreso Internacional sobre Investigación en Didáctica de las Ciencias. Sevilla: Universidad de Sevilla.

Sanmartí N. (2007) Hablar, leer y escribir para aprender Ciencias Sociales. En P. Fernández (coord.). La competencia en comunicación lingïística en las áreas del currículo. Colección Aulas de Verano. Madrid: MEC.

Segura A. (2014) Divulgación científica en las radios universitarias españolas. EDMETIC. Revista de Educación Mediática y TIC 3(1), 44-60.

Villamizar G. (2002) De la escuela en la radio a la radio en la escuela. Sapiens: Revista Universitaria de Investigación 3(1), 57-66. 